\title{
ROUNDUP360
}

Children's orthopaedics

Improving stress distribution in dysplastic hips

While the triple, or Tönnis, osteotomy is well described there is little basic science research surrounding the biomechanical effects. This is all the more surprising given the popularity of the technique; Dr Tönnis' department alone have performed nearly 4000 osteotomies since their technique was first described. Here at 360 , we are firm believers that research into how successful treatments might work is just as important as research into disease mechanisms and novel treatments. A thorough understanding of current treatments might inform understanding of disease and evolution of new techniques. Researchers in Belgrade (Serbia) hypothesised that the changes in contact stresses associated with a Tonnis osteotomy were in the most part responsible for the success of the technique, and designed an ingenious study to investigate this. They used radiographs of a historical series of 75 patients who had undergone Tönnis osteotomy to aid the construction of a $3 \mathrm{D}$ hip model. This was used to calculate a range of biomechanical parameters, including resultant hip force, force inclination, stress pole position peak contact stress and peak contact stress normalised to bodyweight. The authors noted that centre-edge angle increased by $17.85^{\circ}$ postoperatively while the resultant hip force decreased by only $3 \%$. The most significant changes occurred with stress pole position which was shifted medially by nearly $30^{\circ}$ and peak contact hip stresses which decreased by $56 \%$. The authors noted that these biomechanical changes were clinically related to a decrease in abnormal gait patterns.' It is fundamental to understand how current treatments work in order to develop new, more effective treatments. This cunning study leads us at 360 to agree with the authors; the majority of the effect of the Tönnis triple osteotomy likely lies in the improvement in peak stress distribution across the acetabular cartilage, explaining the basis for the reduced rate of degenerative change observed clinically. While this is not groundbreaking, the study is elegant and quantifies stress distributions that were previously only assumed.

\section{The dangers of fashion}

- Fashion is ubiquitous in every walk of life and fashions determine orthopaedic practice more than perhaps we would like to believe. In the wake of a global euphoria surrounding femoral acetabular impingement, sports medicine and the range of new or rediscovered diagnoses, there is the potential for the normal ossification process in children's hips to be mistaken for a pathological process. A research team in New York (USA) sought to distinguish the normal from the abnormal given that the ossification and development pattern of the posterior acetabular wall is unknown and that there is the potential for children to be incorrectly diagnosed with acetabular pathology. They designed a study to establish the normal ossification of the children's acetabulum. They assessed 180 MRI scans taken in patients aged between four and 15 years. All MRIs were evaluated to determine ossification patterns of both the acetabulum and triradiate cartilage. They were examined by a specialist musculoskeletal radiologist and reference made to available radiographs. The chief finding of this study was that the posterior acetabular wall ossifies later than the anterior throughout the child's development. Across the 180 scans, the posterior wall started ossification at eight years with a subsequent discrete rim of posterior calcification at 12 years. These changes occur prior to fusion of the posterior acetabular wall to the pelvis and closure of the triradiate cartilage in all cases. As would be expected, males demonstrated fusion of the posterior wall just over a year later than females. ${ }^{2}$ This superb paper deserves to reach a wider audience than just the paediatric orthopaedic surgeon. Here at 360 , we commend the authors for a fantastic paper which beautifully describes the normal ossification process in the posterior rim of the acetabulum. This paper will help prevent over-intervention.

\section{The natural history of supracondylar fractures}

- Although it is common practice in many centres to treat Gartland type Il fractures with manipulation and Kirschner wiring, there is mixed evidence to support operative treatment of grade II supracondylar fractures.
Researchers from Madrid (Spain) designed a study with the aim of establishing the natural history of unreduced supracondylar fractures. They reason that without understanding the effects of non-operative treatment it is difficult to establish whether intervention is required. They performed a retrospective evaluation of service (Level IV evidence) by evaluating the outcomes of their non-operatively treated patients with a diagnosis of Gartland II supracondylar fractures. The authors used the medical charts and radiographs of 46 consecutive patients with a diagnosis of type II supracondylar fracture. They collated data on the patient (age, gender), injury (hand dominance, side involved, Baumann angle, carrying angle), treatment (period of immobilisation, complications) and outcomes (Mayo Elbow Performance Score and QuickDASH). A total of 46 consecutive patients who were on average 5.5 years old at the time of fracture were identified. Patients presented with an initial mean humeralcapitellar angle of $13^{\circ}$ and Baumann angle of $12^{\circ}$. At final follow-up the injured arm achieved an average $7^{\circ}$ loss of flexion and extension and a mild cubitus varus $\left(9^{\circ}\right.$ versus $\left.12^{\circ}\right)$. Both of these differences were statistically significant, but in reality unlikely to be clinically significant. The clinical outcome scores were also excellent with patients achieving QuickDASH scores of 10 and 96 on the Mayo Elbow Performance Score. However, the Flynn criteria would only grade these results satisfactory in $80.4 \%$ of 
the patients. Although the researchers do not distinguish between Gartland type IIA and IIB fractures, the mean Baumann angle and carrying angle are suggestive that the majority of these patients had type IIA fractures. ${ }^{3}$ Here at 360 , we would regard these results as clinically excellent with insignificant changes in movement arcs and no functional losses in most patients. A counter argument is made in the commentary in the same issue of the JBJS (Am), summing up with this statement: 'Practically, this study indicates that the natural history of the type-Il fracture is not entirely benign. For children, with the exception of toddlers, treatments that involve reduction and pin fixation should likely be recommended.' 4 It does appear that as with most things this paper is very much open to interpretation!

\section{Ankles perform well as knees}

- The Van Nes rotationplasty is one of the more imaginative (and challenging) operative procedures in orthopaedics. The foot and ankle are rotated with the whole lower limb to allow the ankle to sit in a position where it can act as a knee. The patient then wears a prosthetic limb with their own foot sitting inside. Although not commonly performed, the procedure is widely regarded as a good option for patients with proximal femoral deficiency as it removes the need for an above knee prosthesis. Like many unusual procedures for rare diagnoses, the evidence base for this treatment is far from complete and surgeons in Chicago (USA) have reported a long-term prospective case-matched series (Level II evidence) to attempt to establish the long-term functional outcomes of such a procedure. The authors report on 12 patients at a mean of nearly 22 years' follow-up (11 to 45), all of whom completed a range of outcome scores for pain (Faces Pain Scale), limb function (Harris Hip score), back pain (Oswestry score) and prosthetic function. The study participants underwent physical examination, gait analysis, 'up and go' test and computerised posture evaluation. The researchers identified (as one would expect) significant differences in all gait parameters, but amazingly no differences in quality of life scores (SF-36). ${ }^{5}$ The Van Nes procedure is a salvage operation, and we were quite frankly 'gobsmacked' at how well these patients have done.

Although the authors report that patients who have undergone a Van Nes continue to limp and have slight abnormalities in posture, it is difficult to anticipate that any intervention would result in complete resolution of these issues in PFFD. The excellent results reported sup-

port the ongoing use of the Van Nes proceedure.

\section{Intra-articular hip pathology at osteotomy}

- It might seem intuitive that intra-articular hip pathology occurs simultaneously with acetabular pathology in the majority of dysplastic hips, however, surprisingly rarely in general orthopaedic practice are both addressed at the same time, or even the intra-articular hip joint examined. Researchers in Salt Lake City (USA) sought to determine the incidence of intra-capsular pathology in patients undergoing peri-acetabular osteotomy for dysplastic femoral heads. The authors designed a retrospective prognostic study (Level III evidence) with the intention of establishing the incidence of intra-articular hip pathology, and if synchronous, whether treatment of both problems would affect the patient outcome. They retrospectively assessed a comparative case series of 190 patients who underwent peri-acetabular osteotomy (PAO) for dysplasia of the hip. Of these, the first 39 cases performed by the surgeons did not have an arthrotomy, where in the subsequent 151 patients the standard of care changed, and an arthrotomy was routinely performed. Outcomes were assessed with Harris Hip score (HHS) and Tönnis grade. The aim of this study was to establish the prevalence of intra-articular pathology at the time of PAO, and if treatment of concomitant hip pathology negatively affects the outcomes of PAO. The authors noted that $89 \%$ of the capsulotomy group had significant intracapsular pathology requiring treatment, including cam lesions, labral pathology and loose bodies. In this series there was a lower failure rate in the arthrotomy cohort (5.3\% failure) when compared with the PAO alone group (17.9\%). ${ }^{6}$ At first glance we were bowled over, here at 360 , by the strikingly different results between these two cohorts. However, a more careful read is definitely warranted. There are some potentially significant confounders between the two groups. The degree of dysplasia was higher and the patients were treated earlier in the surgical learning curve in the first cohort. That said, this paper is very useful for quantifying the high rates of intra-articular pathology. Surely addressing this at the time of surgery is warranted? We wonder if based on this data, there may be a role for a concomitant arthroscopic approach.

\section{Removal of flexible nails is safe}

- There are conflicting opinions on the timing of paediatric elastic nail system (ENS) nail removal, or even whether to remove them at all. The American Academy of Orthopaedic Surgeons (AAOS) were unable to include a commentary on the removal of them when used in the treatment of paediatric femoral shaft fractures, mostly due to a lack of evidence, in their position statement. The longer they are left the less likely the chance of refracture, but the more difficult removal becomes as the nails become firmly embedded in the bone. Researchers from Honolulu (USA) designed this retrospective cohort study with the intent of plugging the evidence gap uncovered by the AAOS position statement. They designed a retrospective cohort study to determine the outcomes of patients following removal of the elastin nails. A complete chart and radiological review was undertaken and demographic details recorded to assess influence on surgical outcome. The study team identified 163 patients (133 male) who had undergone ENS nailing for femoral fracture (a mixture of Titanium elastic nails and Enders rods) over a five-year period in a single institution. Patients had a mean age of nine years and a mean weight of $34 \mathrm{~kg}$ (14 kg to $139 \mathrm{~kg}$ ). The patients underwent their ENS nail removal at a mean of one year following surgery (2.4 to 63.8 months). There were no significant intra-operative complications although there were four (2.5\%) minor complications. All of these were due to rod migration or bony overgrowth and seen mostly in patients with nails in situ for more than 60 months. There were no cases of refracture and a superficial infection rate of $2 \% .^{7} \mathrm{~A}$ simple paper designed to answer a simple question and the authors have done so with a resounding 'yes'; it is safe to remove ENS nails of both stainless steel and titanium varieties after treatment of a femoral fracture. The complication rates are low, and given the advantages of removing metalwork that will migrate to a completely intramedullary position, we will be ensuring early removal of all of our ENS nails at 360 in future.

\section{Putting the joy into supracondylar fracture fixation}

- Fixing a displaced, pulseless supracondylar fracture remains a challenge for us at 360 , regardless of how many 'sweaty palm' calls for assistance we receive from residents. 
While the Gartland grade II fractures reduce against a periosteal hinge, the grade III fractures do not always do so. In a short paper, surgeons in Aurora (USA) describe a simple technique to add to the armamentarium. They describe the use of a joystick technique to aid reduction. The joystick is passed into the distal fragment on the lateral side, an assistant then applies traction in $90^{\circ}$ of flexion, and the fragment is manipulated with the joystick wire and once reduced, held with several lateral sided wires. The authors describe the technique and the results of eight patients in whom they have used the technique. The patients had a mean age of 7.6 years at injury and underwent joystick-assisted closed reduction and percutaneous pinning. Outcomes were assessed with Flynn's criteria and radiographs (both of the affected and contralateral elbow). At a final follow-up of one year, there were no detectable differences, either radiologically or according to Flynn's criteria, between the affected and contralateral elbow with no recorded iatrogenic complications. ${ }^{8}$ This is a useful little paper to print out and tuck in the box in the operating room for that 'sweaty palm' moment when the whole operating room is silent, looking at you, and try as you might the fracture just won't reduce into a reasonable position.

\section{Talipes: the French way}

- Throughout many developed (and more and more undeveloped) nations, the Ponseti method for treating congenital idiopathic clubfeet has been a revolution. The results are unquestionably excellent with a low surgical intervention rate and a treatment regime so simple it can be used in any healthcare setting. The success of the Ponseti method, and particularly the enthusiasm with which its disciples have evangelised the paediatric orthopaedic world, has eclipsed another conservative management regime: the functional 'French' method. The French regime is a combination of functional splinting and stretching, supplemented with surgical release if required. Surgeons in Nice (France) report a retrospective study of children with idiopathic clubfeet who were treated using the French method.

Patients were classified according to the Dimeglio method and outcomes were assessed using the Ghanem and Seringe score. The authors report on 167 patients, managed with daily physiotherapy and splintage with a progressive regime stopping in childhood. Of the 187 feet, $85(45.5 \%)$ required a supplemental surgical release to address residual deformity. The surgical release involved a complete posterolateral and medial release, tibialis anterior lengthening and occasionally a bony lateral procedure (in cases of forefoot adduction). With an impressive 14.7 year final follow-up, the authors report $98 \%$ good or excellent results. They noted that severe deformity at presentation correlated with poorer functional scores and a higher likelihood of surgery being required. Those requiring surgery had a mean age of 2.5 years and poorer outcomes. However, by final followup, results were good or excellent in $95 \%$ of cases. The authors start their paper commenting that there are two main options for treating talipes. While they have adequately demonstrated that the French method can be used to good effect, their patients required a major surgical release almost $50 \%$ of the time and major input from physiotherapists. ${ }^{9}$ This echoes a much smaller comparative study ${ }^{10}$ with shorter follow-up which demonstrated higher surgical intervention rates with the French method but similar functional results. It seems that similar results can be achieved with lower rates of surgical intervention with the Ponseti method.

\section{REFERENCES}

1. Vukasinovic Z, Spasovski D, Kralj-Iglic V, et al. Impact of triple pelvic osteotomy on contact stress pressure distribution in the hip joint. Int Orthop 2013;37:95-98.

2. Fabricant PD, Hirsch BP, Holmes I, et al. A radiographic study of the ossification of the posterior wall of the acetabulum: implications for the diagnosis of pediatric and adolescent hip disorders. J Bone Joint Surg [Am] 2013;95-A:230-236. 3. Moraleda $L$, Valencia $M$, Barco $R$, González-Moran G. Natural history of unreduced Gartland type-II supracondylar fractures of the humerus in children: a two to thirteen-year follow-up study. J Bone Joint Surg [Am] 2013;95-A:28-34.
4. Blasier RD. Gartland type-II supracondylar humeral fractures in children: commentary on an article by Luis Moraleda, MD, et al. "Natural history of unreduced Gartland type-II supracondylar fractures of the humerus in children: a two to thirteen-year follow-up study". J Bone Joint Surg [Am] 2013;95-A:e7-e7.

5. Ackman J, Altiok H, Flanagan A, et al. Long-term follow-up of Van Nes rotationplasty in patients with congenital proximal focal femoral deficiency. Bone Joint J 2013;95-B:192198.

6. Ginnetti JG, Pelt CE, Erickson JA, Van Dine C, Peters CL. Prevalence and treatment of intraarticular pathology recognized at the time of periacetabular osteotomy for the dysplastic hip. Clin Orthop Relat Res 2013;471:498503.

7. Levy JA, Podeszwa DA, Lebus G, Ho CA, Wimberly RL. Acute complications associated with removal of flexible intramedullary femoral rods placed for pediatric femoral shaft fractures. $J$ Pediatr Orthop 2013;33:43-47.

8. Novais EN, Andrade MA, Gomes DC. The use of a joystick technique facilitates closed reduction and percutaneous fixation of multidirectionally unstable supracondylar humeral fractures in children. J Pediatr Orthop 2013;33:14-19.

9. Rampal V, Chamond C, Barthes X, et al. Long-term results of treatment of congenital idiopathic clubfoot in 187 feet: outcome of the functional "French" method, if necessary completed by soft-tissue release. J Pediatr Orthop 2013;33:48-54.

10. Steinman S, Richards BS, Faulks S, Kaipus K. A comparison of two nonoperative methods of idiopathic clubfoot correction: the Ponseti method and the French functional (physiotherapy) method: surgical technique. J Bone Joint Surg [Am] 2009;91-A(Suppl 2):299-312. 